\title{
ENVEJECIMIENTO Y POLÍTICA: UN DEBATE POLITOLÓGICO
}

\author{
Ageing and Politics: A debate in political science
}

\author{
CRISTINA ARES CASTRO-CONDE \\ Universidad de Santiago de Compostela \\ cristina.ares@usc.es
}

\author{
Cómo citar/Citation \\ Ares Castro-Conde, C. (2018). \\ Envejecimiento y política: un debate politológico. \\ Revista de Estudios Políticos, 179, 171-198. \\ doi: https://doi.org/10.18042/cepc/rep.179.06
}

\section{Resumen}

En un nuevo contexto de escasez de recursos públicos y de debate acerca de su equitativa y eficiente distribución, este trabajo define tres marcos discursivos elementales sobre la relación entre el proceso de envejecimiento de la población y el Estado de bienestar: el marco tradicional welfarista de protección de la vejez, el discurso de la guerra entre generaciones y el marco de la nueva solidaridad intergeneracional. Se subraya cómo el discurso de la guerra entre generaciones en su modalidad neoliberal busca erosionar el modelo social europeo. Asimismo, se identifican elementos diferenciadores entre este discurso neoliberal y un segundo marco emergente construido también en torno a la idea de ruptura del pacto intergeneracional, pero progresista. Finalmente, se seńalan temas para el desarrollo de la incipiente agenda de investigación en ciencia política sobre los efectos del envejecimiento.

\section{Palabras clave}

Envejecimiento; Estado de bienestar; modelo social europeo; justicia/equidad generacional; guerra entre generaciones; solidaridad intergeneracional; envejecimiento activo.

\section{Abstract}

Within a new context of public resource scarcity and debate on their fair and efficient allocation, this article defines three primary discourses framing the relationship 
between population ageing and the welfare state: the classical welfarist frame of protection of the elderly; the 'war between generations'; and the 'new intergenerational solidarity'. It highlights how the neoliberal discourse of the war between generations seeks to erode the European social model. Subsequently, it sheds light on some elements differentiating this neoliberal frame from an emerging 'progressive' version of the idea of the collapse of the intergenerational contract. Finally, it pays attention to issues that could contribute to the emerging agenda in Political Science on the effects of population ageing.

\section{Keywords}

Ageing; welfare state; European social model; generational justice/equity; war between generations; intergenerational solidarity; active ageing. 
I. INTRODUCCIÓN. II. ESTUDIOS DE LOS EFECTOS POLÍTICOS DEL ENVEJECIMIENTO. III. LOS MARCOS DISCURSIVOS DEL ENVEJECIMIENTO EN RELACIÓN CON EL ESTADO DE BIENESTAR. IV. CONCLUSIONES. Biblografía. Anexo. Glosario.

La imagen de la tercera edad como un bloque de votantes movidos por los «intereses de los séniores» es un hombre de paja que se mantiene porque sirve a determinados propósitos (Schulz y Binstock, 2006: 208).

Los mayores no son las únicas fuerzas sosteniendo el statu quo de los sistemas de pensiones, no siempre están en contra de su reforma, y cuando lo están no siempre ganan (Lynch, 2015: 58).

Las democracias que encanecen (greying democracies) no son per se ni mejores ni peores democracias (Goerres, 2009: 174).

\section{INTRODUCCIÓN}

El envejecimiento de la población es un proceso demográfico propio de las sociedades desarrolladas, de alcance global, que consiste en el aumento del porcentaje de personas de 65 años o más sobre el total de la población mundial y de cada uno de los países desarrollados. Se ha escrito que el envejecimiento está provocando el cambio social más importante desde la universalización de la educación básica en las democracias avanzadas. Esta transformación afecta a un número creciente de individuos de todas las edades a nivel micro y macro, personas que se relacionan durante más años con mayores, en los distintos roles que estos desempeñan, en la familia, como consumidores, destinatarios de políticas públicas u otros.

En la génesis del envejecimiento se encuentra fundamentalmente un factor positivo: el incremento de la esperanza de vida al nacer, debido, entre otras causas, a mejoras en la calidad de vida y el cuidado de la salud. La crisis de fertilidad, o el descenso en las tasas de natalidad, también suele jugar un papel.

Llamo la atención, en primer lugar, sobre la necesidad de distinguir entre el cambio demográfico y sus consecuencias sociales y políticas, así como destaco el hecho de que esta transformación se produce de forma simultánea a otros 
cambios con los que interactúa. No se están materializando las expectativas de convergencia en torno al modelo de envejecimiento occidental en un escenario internacional globalizado (Fennell et al., 1988), sino que emergen nuevas formas sociales y políticas de envejecimiento a nivel internacional, nacional, regional y local (Held y McGrew, 2002).

Por tanto, solo tras realizar estudios en perspectiva comparada, se puede analizar el impacto del envejecimiento en la democracia y, en su caso, señalar implicaciones normativas. En cambio, la discusión pública actual sobre un tema tan relevante resulta en exceso superficial y se halla trufada de eslóganes sensacionalistas.

Este trabajo desea contribuir a la discusión sobre los efectos políticos del envejecimiento, definiendo tres marcos discursivos básicos sobre envejecimiento y políticas de bienestar: 1) el marco welfarista clásico de protección de la vejez, 2) el discurso emergente y popular en los medios de comunicación sobre el conflicto o la guerra entre generaciones, a veces también llamado de la equidad o la justicia generacionales, y 3 ) el más reciente marco de la nueva solidaridad entre generaciones, impulsado por las instituciones de la Unión Europea (UE) (Comisión Europea, 2005).

Asimismo, identifico temas para el estudio comparado desde la ciencia política de las dinámicas de las sociedades envejecidas. En particular, para el examen del impacto del cambio demográfico en la política (politics) en Europa, el continente más envejecido. En 2016, el porcentaje medio de población de 65 años o más en los países de la UE era del 19,2\%, y la esperanza de vida al nacer de hombres y mujeres de 77,9 y 83,3 ańos, respectivamente ${ }^{1}$.

Además de las conclusiones, las referencias y un breve glosario, estructuro el texto en dos epígrafes sustantivos. El apartado segundo revisa las investigaciones politológicas y de otras disciplinas sociales sobre los efectos políticos del envejecimiento. A continuación, el tercer epígrafe define los tres marcos discursivos elementales antes referidos que relacionan el cambio demográfico y las políticas de bienestar. Se pone el foco en la naturaleza de hombre de paja del discurso neoliberal de la guerra entre generaciones, que también se diferencia en el plano teórico de un segundo marco emergente en torno a la idea de ruptura del conflicto intergeneracional, pero progresista.

1 La tabla del anexo contiene estos datos demográficos por Estado miembro, junto con (entre paréntesis) el indicador ańos de vida saludable al nacer, definida como la ausencia de limitaciones a la autonomía personal. 


\section{ESTUDIOS DE LOS EFECTOS POLÍTICOS DEL ENVEJECIMIENTO}

Hasta el año 2003, la ciencia política apenas había hecho contribuciones al análisis del envejecimiento. Si bien sobre políticas públicas (policies) las aportaciones, tanto de la nuestra como de otras disciplinas afines, son más abundantes, los efectos del envejecimiento en la política (politics) continúan siendo un terreno apenas explorado.

Debe reconocerse que el envejecimiento constituye un objeto particularmente adecuado para ser abordado conjuntamente por parte de distintas áreas de conocimiento. De hecho, con este propósito, a partir de la década de 1960, se ha ido desarrollando un nuevo campo de estudio, la gerontología, que se define a sí misma como ciencia. A partir del énfasis inicial en asuntos biológicos y biomédicos, los gerontólogos han ido ampliando su abanico de temas incorporando cuestiones antropológicas, sociales, económicas, políticas, e incluso históricas y artísticas, entre otras (Bengtson et al., 2005; Kolb, 2014).

Si bien algunos investigadores han rechazado la necesidad o aceptado la imposibilidad de imponer un único marco teórico sobre el envejecimiento, desde la gerontología se ha tratado de ofrecer teorías generales de este proceso, como la teoría de la desvinculación (véase más abajo) o las teorías de la actividad, los roles o el intercambio ${ }^{2}$.

La literatura ha alertado extensamente sobre la falta de base empírica de la idea de gerontocracia, grey o senior power (Schulz y Binstock, 2006; Walker, 2006), esto es, de la creencia según la cual, conforme aumenta el número de mayores, también lo hace su influencia en el proceso político, que sería ejercida con el propósito de defender unos supuestos intereses comunes de los séniores. Se ha comenzado también a deconstruir el proceso de neoliberalización de la vejez (Macnicol, 2015), o las implicaciones de la pérdida de importancia del factor edad en las sociedades postmodernas, así como otras asunciones del marco de la guerra entre generaciones que detallaré en el apartado siguiente.

Walker (2006) ha distinguido entre nuevas y viejas políticas de envejecimiento. Las primeras atribuyen a los mayores un rol pasivo, casi limitado a la percepción de pensiones y otras prestaciones públicas. Las viejas políticas alimentan los estereotipos negativos asociados a cumplir años - la imagen de los mayores como «pobres, frágiles, dependientes socialmente, objetos de discriminación y, sobre todo, meritorios (deserving)" (Binstock, 1991: 1)—. La propia academia ha contribuido a alimentar estos estereotipos con teorías como la funcionalista de la desvinculación (disengagement). Según esta teoría,

2 Sobre el desarrollo de los estudios gerontológicos véase, por ejemplo, Kolb (2014: 14-17). 
la tercera edad consiste inevitablemente en un proceso de desconexión del individuo del resto de la sociedad (Cumming y Henry, 1961; Westerhof y Tulle, 2007).

Las nuevas políticas de envejecimiento, en cambio, promueven la participación social y política de los mayores (Vera-Sanso et al., 2014; Walker, 2014). Su aparición se produce, en una primera ola, a mediados de la década de 1970, con la crisis fiscal provocada por el shock en el precio del petróleo, y en una segunda ola en los años 1980. Fue entonces cuando los costes de los cuidados de larga duración y la maduración de los sistemas de pensiones comenzaron a enmarcarse como una "carga" (burden), y la "ratio de dependencia» entre mayores y trabajadores en activo empezó a emplearse como base empírica del discurso apocalíptico del colapso del sistema de bienestar.

Aunque las nuevas políticas de envejecimiento no siempre son contrarias a la responsabilidad pública sobre el bienestar de los séniores, no debe olvidarse que la explicación del tránsito inicial de las viejas a las nuevas políticas de envejecimiento es la voluntad de reducir la intervención del sector público.

La definición del envejecimiento como un problema financiero presta un gran servicio a quienes defienden objetivos neoliberales (Minkler y Robertson, 1991; Quadagno, 1989; Schulz y Binstock, 2006; Vincent et al., 1996; Walker, 1990). Los primeros recortes en el gasto público en pensiones y servicios sociales fueron adoptados en la década de 1980 en Estados Unidos y el Reino Unido, sencillamente, debido al éxito de una nueva derecha neoliberal revisionista de las políticas conservadoras, la cual incluso ha tratado de imponer sus asunciones globalmente a través de organizaciones internacionales como el Fondo Monetario Internacional, el Banco Mundial, más recientemente, la Organización Mundial del Trabajo y, en menor medida, la Organización para la Cooperación y el Desarrollo Económico (Walker, 2006: 343).

Nótese igualmente la creciente importancia de los aspectos globales de las nuevas políticas de envejecimiento (Walker, 2006). Además de aportar proyecciones demográficas, entidades como el Banco Mundial (1994), Naciones Unidas (2003; Sidorenko y Walker, 2004), la Organización para la Cooperación y el Desarrollo Económico (1988, 1998 y 2001) o la Organización Mundial de la Salud (2002) han promovido medidas sobre "calidad de vida» en la tercera edad y, en general, políticas globales de envejecimiento, con impacto variable en distintos contextos.

Por otra parte, es preciso subrayar las contribuciones de Lynch (2006 y 2015) al estudio del envejecimiento desde la ciencia política. Entre sus resultados destacan los siguientes. En primer lugar, la variación en la maduración del sistema de pensiones y el envejecimiento de la sociedad no han explicado en el pasado ni la extensión ni el apoyo a las pensiones (Lynch, 2006 y 2015). 
La importancia electoral de los mayores se produce, sobre todo, una vez que las pensiones ya han sido introducidas (Lynch, 2015: 51). La puesta en marcha de extensas medidas de apoyo a las personas mayores en los sistemas de bienestar durante el siglo pasado no está relacionada con la presión de los beneficiarios, ni como votantes ni a través de grupos de interés o partidos de pensionistas. Tiene que ver, en cambio, con las preferencias de los entonces trabajadores en activo, empresarios, entidades promotoras de la reducción de la pobreza con apoyos en la opinión pública, algunos políticos y sindicatos.

Además, el tipo de competición política (más o menos programática o particularista) tiene un peso importante en la explicación del porcentaje del gasto social destinado a los mayores en los distintos países de la OCDE. Por ejemplo, en Austria, Bélgica, España, Estados Unidos, Grecia, Italia y Japón, donde muchos políticos no han seguido líneas de competición programáticas, el gasto en pensiones aumentó más aceleradamente. La competición particularista tiende a evitar la conversión en universalistas de los sistemas de pensiones ocupacionales. Incluso, cuando en un contexto predomina claramente la competición programática, la colonización de las instituciones por parte de políticos clientelares hace menos probable la reforma de los modelos ocupacionales, porque las preferencias de los políticos partidarios de un modelo universal se acomodan al contexto.

Otra de las grandes aportaciones al estudio de los efectos políticos del envejecimiento es la de Goerres (2007a, 2007b, 2008 y 2009). Este autor insiste también con acierto en la necesidad de emplear enfoques comparativos para el análisis de las dinámicas políticas de las sociedades envejecidas. Uno de sus principales argumentos para recomendar esta visión comparada es la naturaleza de los efectos de cohorte, esto es, de las características compartidas por las personas nacidas durante un determinado período temporal. A diferencia de los efectos generacionales, que son rasgos vinculados a la edad cronológica sin más, los efectos de cohorte varían entre países/contextualmente. En términos de niveles de educación, socialización política, valores más o menos postmaterialistas, por ejemplo, no es lo mismo haber nacido en la década de 1940 en Madrid o en París. Al mismo tiempo, cada sociedad construye su propia imagen de la vejez (Goerres, 2009: 35).

Las grandes preguntas de investigación de Goerres han sido cómo y por qué la participación individual de los mayores diverge de la de los jóvenes, y en qué medida estas diferencias importan en el proceso democrático de las sociedades envejecidas. Aunque, incluso sobre estos aspectos se observan variaciones entre países, hay dos formas de participación en las que es más probable que los mayores se impliquen más que los jóvenes: la militancia en un partido y el voto. La militancia de los séniores se ve favorecida por un efecto generacional: el haber vivido más años en la misma localidad. También, 
algunos países, como Alemania, han institucionalizado estructuras organizativas para que las personas mayores se encuentren mejor representadas y, frente a la tendencia general de declive en la militancia partidaria en Europa Occidental, este tipo de afiliaciones aumenta.

Además, en la década de 1980, sobre todo como respuesta a los primeros recortes en las prestaciones públicas percibidas por los mayores, comenzaron a aparecer en Europa partidos sénior (senior o grey parties). Estos partidos han tenido poco éxito, quizá en parte por la adaptación de la oferta programática de los partidos tradicionales, cuestión pendiente de verificar y destacable en la agenda de investigación de la ciencia política.

El principal hallazgo de Goerres es que en sociedades con actitudes positivas hacia las personas mayores, incluso con una ratio de dependencia preocupante, si su protección pública es adecuada, resulta poco probable no solo que los mayores tengan intención de tomar el control del proceso político, sino incluso que lleguen a activarse a través de cualquier forma de participación política (Goerres, 2009: 18).

Respecto al voto, en sistemas de bienestar público generosos con la tercera edad, el impacto del envejecimiento en los resultados electorales ha sido irrelevante. En cambio, en las democracias con sistemas electorales proporcionales, donde los partidos pequeños pueden fácilmente obtener representación, los jóvenes sí tienen un incentivo para diferenciar el voto del de sus mayores, ya que estos últimos, en principio, tienden a seguir votando a partidos grandes y de gobierno. No obstante, el sesgo de los séniores a favor de los partidos tradicionales no es general, y cabe esperar que desaparezca, por la pérdida de relevancia de la socialización política temprana, en sistemas de partidos crecientemente caracterizados por la volatilidad y el desalineamiento.

No puedo dejar de destacar que los datos de los que se dispone hasta este momento son claramente contrarios a la creencia, recurrente en el actual debate público, superficial e ideológicamente sesgado, sobre los efectos políticos del envejecimiento, de que las opciones electorales de los mayores son más conservadoras en términos económicos.

En cambio, en las sociedades que podrían etiquetarse como amigables con los séniores, en las que estos se sienten respetados y protegidos y no tienen incentivos para la movilización política, sí se observa una mayor actividad política de los jóvenes, a fin de equilibrar el reparto del gasto social entre generaciones, en principio, evitando que crezca el tamańo del porcentaje de recursos públicos destinado a los mayores.

La evidencia empírica de Goerres es consistente, por otra parte, con estudios anteriores que desmienten la existencia de una identidad común de la tercera edad (Day, 1990; Binstock, 1972), que podría haber favorecido el ejercicio de la influencia a través de la acción colectiva. 
Los séniores, no obstante, podrían llegar a activarse políticamente en defensa de intereses individuales comunes, si las pensiones y otras prestaciones públicas que les benefician sufriesen recortes que pusiesen en riesgo su bienestar. De hecho, los cambios que se han ido introduciendo en los sistemas de pensiones (Natali, 2012; Doyle, 2015; Williamson y Béland, 2016) suelen prever un retraso en su puesta en marcha, a fin de evitar la contestación y un eventual castigo electoral de los séniores.

Sin embargo, no parece probable que se desarrolle una conciencia política de grupo en los mayores. La clase social, el género, el lugar de residencia, entre otros factores, son más importantes que la edad cronológica en su identidad individual. Los séniores rara vez se muestran más solidarios con las personas de su generación que con el resto de la sociedad.

Se constatan, además, otras limitaciones para la activación política de las personas mayores. Por ejemplo, estas pueden haber aprendido que determinadas formas de participación no son eficaces, o entender que a partir de una determinada edad o de su salida del mercado laboral «no deberían tener que activarse» (Goerres, 2009: 157). Goerres interpreta esta evidencia como el impacto de los estereotipos o las imágenes predominantes en la sociedad sobre la jubilación y la tercera edad. Añado que se puede esperar que el marco welfarista tradicional siga predominando en mayor medida entre los séniores, quienes son menos permeables al discurso de la guerra entre generaciones e incluso al de la nueva solidaridad intergeneracional. Los mayores pueden tener firmemente interiorizada la creencia de que se han ganado el derecho a despreocuparse de su seguridad.

Finalmente, Goerres (2009: 169), retomando la sugerencia de Cutler (1977), ha propuesto establecer una nueva disciplina llamada "Gerontología política», dedicada al análisis de los aspectos políticos de la tercera edad y del envejecimiento. Como se ha ido mostrando parcialmente en los párrafos anteriores, ha planteado preguntas de investigación sobre el impacto de este cambio demográfico en el funcionamiento de las instituciones y de las organizaciones de la sociedad civil, la calidad de las decisiones públicas y el propio concepto de democracia, que merecerían una mayor atención de la ciencia política.

En el epígrafe siguiente me centraré en la definición de los marcos discursivos básicos sobre envejecimiento y bienestar.

\section{LOS MARCOS DISCURSIVOS DEL ENVEJECIMIENTO EN RELACIÓN CON EL ESTADO DE BIENESTAR}

Como he señalado en la introducción, se debe distinguir entre el fenómeno o el proceso demográfico del envejecimiento y sus consecuencias sociales 
y políticas. El primero, el crecimiento del grupo de personas de 65 años y más, puede enmarcarse como una debilidad o como una fortaleza de una sociedad. Muchos entre quienes apuestan por la lectura negativa tienden a definirlo como un problema presupuestario del sector público, afirman que el gasto destinado a las personas mayores es insostenible, y llegan a sentenciar que los Estados de bienestar tienen fecha de caducidad. El cambio demográfico, desde esta perspectiva, plantea un juego de suma cero en el que las distintas generaciones compiten por recursos públicos escasos y las transferencias intergeneracionales producen necesariamente ganadores y perdedores.

Este punto de vista comienza a imponerse en la conversación pública europea sobre envejecimiento, al ser aceptado un tanto acríticamente por parte de algunos ciudadanos y medios de comunicación con influencia sobre el debate político. Sin embargo, esta no es la única forma de enmarcar el proceso de envejecimiento de la población, ni siquiera el conflicto entre generaciones, no es respetuosa con la evidencia empírica disponible, y tampoco es ideológicamente neutra.

Se ha escrito también sobre los aspectos positivos del envejecimiento como tendencia demográfica en las sociedades desarrolladas (Kolb, 2014), subrayando, por ejemplo, la riqueza que aportan las nuevas cohortes de adultos mayores, en muchos países más instruidos y con experiencias vitales más diversas que en el pasado (Westerhof y Tulle, 2007). Desde este punto de vista, el envejecimiento no se presenta como un juego de suma cero sino de suma positiva en el que todas las generaciones, que se perciben como interdependientes, pueden salir ganando. Así, cuando se enfrentan dificultades para el equilibrio financiero, conviene acordar con antelación reformas que garanticen la sostenibilidad a largo plazo de las pensiones y otros programas de gasto público.

Las propias instituciones de la UE han querido trasladar una visión razonablemente optimista sobre la continuidad del contrato intergeneracional, y han promovido «una nueva solidaridad entre generaciones» (Comisión Europea, 2005), de ahí la etiqueta escogida para mi tercer marco discursivo. Incluso han dedicado el ańo 2012 al envejecimiento activo y la solidaridad intergeneracional.

En este apartado ordeno estas y algunas otras ideas en torno al envejecimiento y las políticas de bienestar en tres discursos, que resumo en la tabla 1: 1) el marco welfarista clásico de protección de la vejez, 2) el discurso de la equidad/justicia generacional o del conflicto/guerra entre generaciones, y 3) el marco de la nueva solidaridad entre generaciones. Los discursos primero y tercero son similares, pero no idénticos. Considero útil distinguirlos conceptualmente porque difieren en su definición de solidaridad así como en su visión, más o menos positiva, de las personas mayores. 


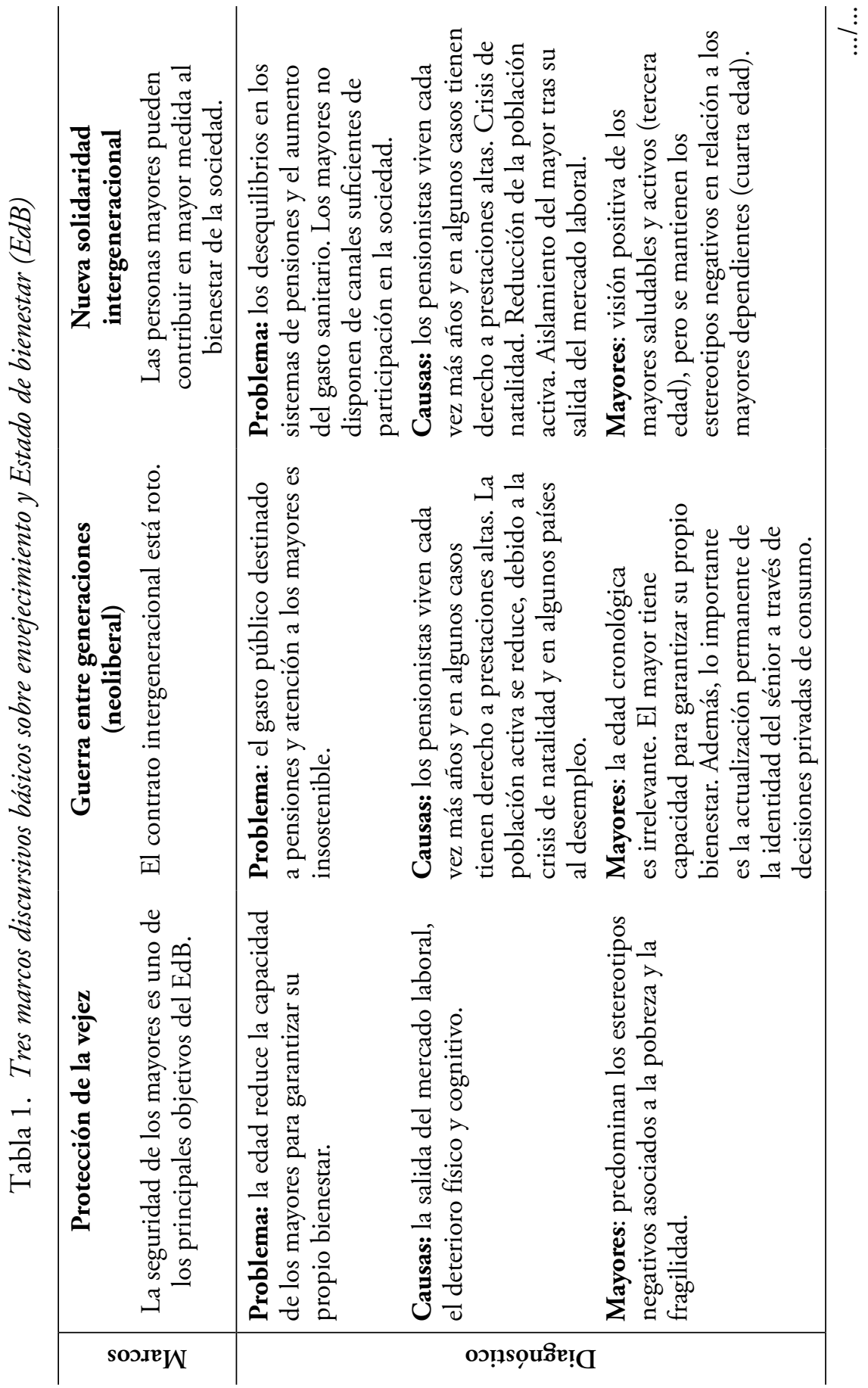




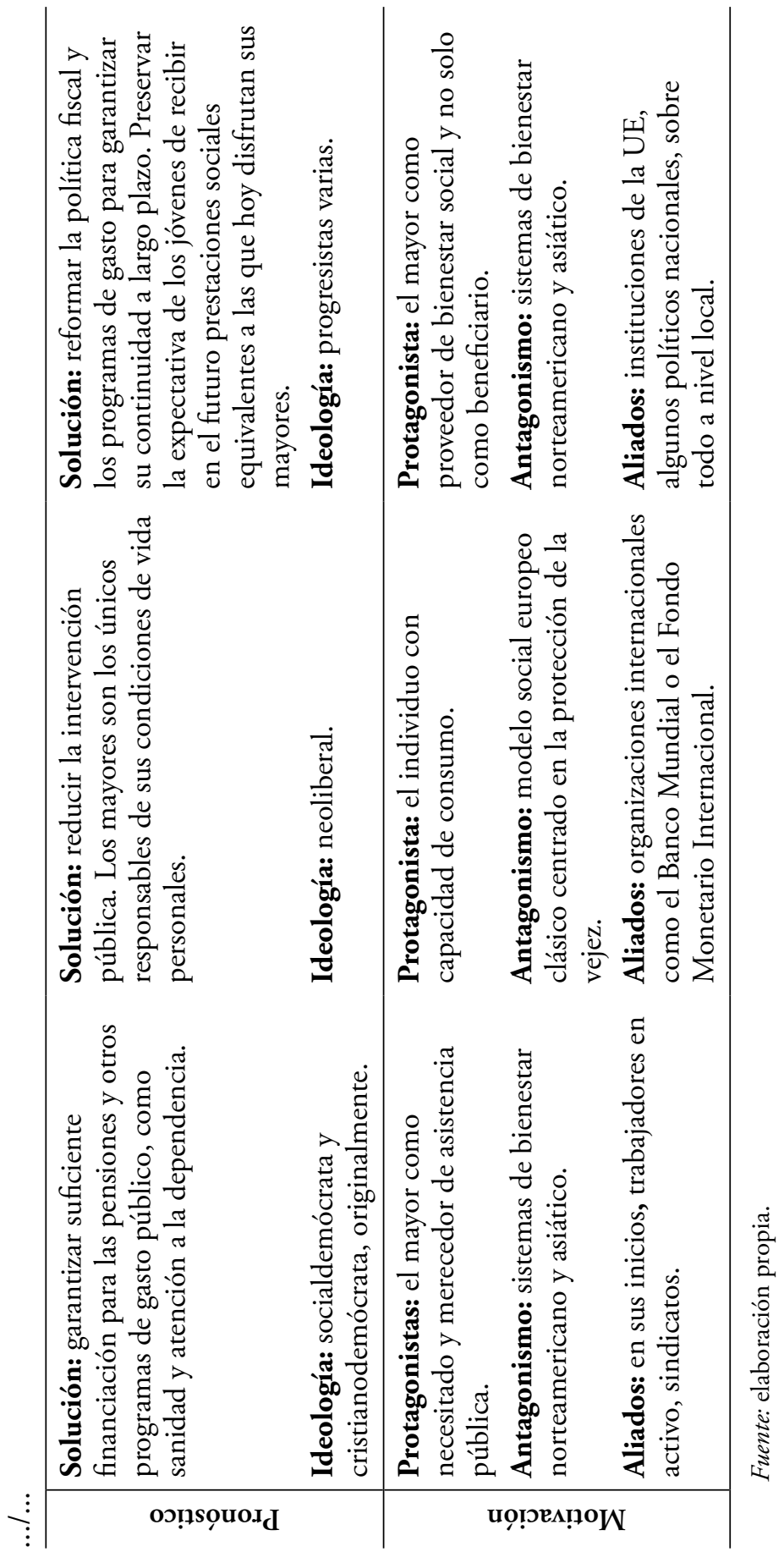


Además, no solo se habla de guerra entre generaciones desde posiciones neoliberales, sino que existen dos marcos emergentes distintos en torno a la idea de caducidad del contrato intergeneracional, que resumo, a continuación, en la tabla 2.

No debe olvidarse que la protección de la vejez es una de las primeras y principales razones de ser del modelo social europeo, en sus diversas concreciones en los distintos regímenes de bienestar (Lynch, 2006; Walker, 2006; Moreno, 2013). Tampoco, que hoy en día los mayores siguen siendo los principales beneficiarios del gasto social, como pensionistas y usuarios de la sanidad pública. En el discurso welfarista original, o marco número uno, los ciudadanos, tras haber contribuido a la generación de riqueza y bienestar durante décadas, una vez abandonan el mercado laboral o cumplen una determinada edad (habitualmente 65 años), entran en una fase de «merecido descanso». Entonces, se encuentran en una posición de mayor vulnerabilidad, durante la cual las generaciones en edad de trabajar deben garantizar su bienestar a través de la financiación de sus pensiones, asistencia sanitaria y otros servicios sociales.

La clave del contrato intergeneracional welfarista reside en que las generaciones más jóvenes, en general, y los trabajadores en activo, en particular, albergan la expectativa de que al alcanzar el umbral etario convenido, disfrutarán de beneficios públicos equivalentes a los de sus mayores.

El tercer discurso de la nueva solidaridad intergeneracional está construido sobre una percepción más positiva de los mayores en comparación con el número uno. Subraya su menor dependencia de las generaciones jóvenes, en muchos casos, hasta una edad más avanzada y muy posterior a la de jubilación. Las mejores condiciones de salud y en algunos casos las experiencias vitales más variadas de las nuevas cohortes de mayores hacen que estas personas puedan seguir contribuyendo en mayor medida que en décadas pasadas al bienestar social. Por ello, se plantea, de una parte, la posibilidad de retrasar la edad de jubilación, y/o de que los séniores participen en la formación de trabajadores junior. De otra, se defiende la conveniencia de poner en valor las aportaciones instrumentales y afectivas de los mayores a sus familias y/o a su entorno social más amplio, por ejemplo, a través de programas de voluntariado.

La nueva solidaridad pone el foco en los años en los que el sénior no solo disfruta sino que también contribuye activamente al bienestar de la sociedad. En cambio, aparece una nueva fase del ciclo vital, la cuarta edad, caracterizada por la pérdida de autonomía o la dependencia, a la que se trasladan los estereotipos negativos antes asociados a todas las personas de 65 años o más. La distinción entre la tercera y cuarta edad no es cronológica. Por una parte, están los mayores saludables y activos, y por otra los enfermos y necesitados de asistencia en sus rutinas diarias. La visión positiva de la vejez del tercer marco está reservada a los primeros: quienes retrasan su jubilación, ayudan a 
personas de su entorno, como a sus hijos en la crianza de los nietos, y consumen bienes y servicios culturales o turísticos, entre otros.

En resumen, el marco de la nueva solidaridad pone más en valor, en comparación con el welfarista tradicional, la experiencia y las vivencias a partir de los 65 años. Pero, a diferencia del discurso de la justicia/equidad generacional, apuesta por mantener el reconocimiento de la singularidad de las últimas etapas del ciclo de la vida. Claro está que en el tercer marco subyace, como en el segundo, el desafío financiero para un país envejecido que supone pagar pensiones más altas durante más años para un porcentaje creciente de mayores sobre el total de la población. Sin embargo, en el tercer marco, por una parte, el cambio en la valoración del mayor no dependiente va más allá de la búsqueda de una solución a un problema de equilibrio presupuestario y, por otra, el principal objetivo es proteger el modelo social europeo, dando continuidad al contrato intergeneracional mediante su adaptación a la nueva realidad demográfica.

Para ello, por ejemplo, la Comisión Europea, principal promotora de la nueva solidaridad entre generaciones, ha propuesto una estrategia llamada "de envejecimiento activo" (active ageing), que se refiere a la extensión de la actividad de los mayores tanto en el mercado laboral como en el ámbito social a través de la participación en actividades cívicas o culturales (Eurostat, 2011). En el plano teórico, la definición de «envejecimiento activo» de esta institución es multidimensional, contemplando cuestiones relacionadas con la seguridad y la salud de los mayores y su participación social y política. No obstante, en la práctica, se ha puesto el foco en la prolongación de la vida laboral y otras medidas orientadas a facilitar los equilibrios presupuestarios, en parte también porque sobre temas de bienestar los Estados miembros apenas han transferido competencias al nivel supranacional ${ }^{3}$.

Apunto, también, que el concepto de envejecimiento activo de la política de la UE diverge del propuesto por la Organización Mundial de la Salud, simplemente porque esta última adopta el punto de vista del mayor al hablar del «proceso de optimizar las oportunidades para la salud, la participación y la seguridad a fin de aumentar la calidad de vida cuando la gente envejece» (OMS, 2002).

3 Entre los retos que plantea el envejecimiento de la población, la Comisión destaca los siete siguientes: presión sobre los presupuestos públicos y los sistemas fiscales; tensión sobre los sistemas de pensiones y seguridad social; ajustar la economía y en particular los puestos de trabajo a una fuerza laboral que envejece; posible escasez de trabajadores en activo; probable necesidad de aumentar el número de profesionales sanitarios con formación; una mayor demanda de servicios sanitarios y cuidados institucionalizados de larga duración; y, finalmente, el conflicto potencial entre generaciones sobre la distribución de los recursos (Eurostat, 2011:7). 
El discurso del envejecimiento activo en sus distintas variantes es más amplio que el paradigma del «envejecimiento exitoso" (successful aging), que se había impuesto en gerontología desde la publicación seminal de Rowe y Kahn (1987). La noción de «envejecimiento exitoso» se centra en los siguientes tres criterios: bajos niveles de enfermedad y dependencia, funcionamiento físico y cognitivo amplio y compromiso activo con la vida (Rowe y Kahn, 1998). Tanto el discurso del envejecimiento exitoso como el del envejecimiento activo han sido objeto de crítica por limitar la visión positiva de la vejez a los mayores sanos y activos y estigmatizar al resto de la población mayor (George, 2006; Higgs y Gilleard, 2015b; Minkler y Fadem, 2002).

Para evitar decir que los mayores discapacitados o simplemente quienes manifiestan un deterioro en sus condiciones físicas y/o cognitivas no pueden tratar de mantenerse activos o envejecer exitosamente, y teniendo en cuenta que la evidencia empírica muestra en muchos casos falta de correspondencia entre las condiciones objetivas y el bienestar subjetivo (George, 2006: 322), al menos deberían incorporarse al concepto las percepciones subjetivas de bienestar.

Subrayo que el envejecimiento exitoso se mantuvo durante décadas como el paradigma dominante en gerontología, sin apenas conectar el bienestar de los mayores con variables económicas, sociales y políticas de nivel macro. Se trata, por consiguiente, de un terreno idóneo para el análisis multinivel.

El segundo discurso sobre envejecimiento y políticas de bienestar se distancia radicalmente del primero y del tercero, desde el momento en que se opone a la idea de diferenciar individuos en función de la edad. La edad cronológica deja de ser un elemento relevante en la definición de la identidad individual, la cual está sujeta a actualización permanente en las decisiones cotidianas de consumo y estilo de vida (Beck, 1992; Giddens, 1991).

La negativa desde este marco a reconocer la tercera edad como una etapa diferenciada de la madurez anterior a los 65 ańos forma parte de un proceso más amplio de desestandarización del ciclo vital en la «sociedad líquida» (Bauman, 2007), donde el envejecimiento es contingente y la vida después de la jubilación incierta, al tiempo que repleta de oportunidades para reinventarse (Higgs y Gilleard, 2015: 128).

Nótese que este discurso, como mucho, describiría la vejez de una élite de jubilados ricos, la cual representaría en torno al $5 \%$ del total de los mayores (Macnicol, 2015: 10). Como en el tercer marco, no hay una visión negativa del sénior saludable y activo, porque no hay estereotipos o discriminación en razón de la edad. Sin embargo, se margina al mayor no productivo, enfermo, inactivo, que se relacionaría con el resto de la sociedad casi únicamente a través del cuidado y sus distintas narrativas y prácticas.

El hiperindividualismo y el consumismo característicos del tránsito de la modernidad a la postmodernidad también afectarían a la atención a las 
personas mayores dependientes. Estas y/o sus cuidadores informales demandarían mayor participación en decisiones asistenciales antes consideradas terreno exclusivo de los profesionales sanitarios o sociales. Del mismo modo, los expertos serían también más receptivos a la implicación de los usuarios en la práctica profesional (Walker, 2006: 346-347).

Este cambio cultural, que puede observarse en distintas sociedades envejecidas, se encuentra, no obstante, con múltiples barreras, como las desigualdades de género o clase (Westerhof y Tulle, 2007). Sin embargo, la transformación cultural trasciende la definición del segundo marco. Desde el tercer discurso también se han abordado iniciativas para la promoción de la cultura del empoderamiento de los mayores, en la que las Administraciones regionales y locales están jugando un papel clave (Walker, 2006: 346; Walker y Nagele, 2009: 19).

Subrayo que la discriminación del mayor no activo en el discurso del conflicto entre generaciones se retroalimenta con la propia visión apocalíptica de este marco que retrata una bolsa de personas frágiles y dependientes, la cual no deja de crecer, y con ella el coste de su atención sanitaria y social para el resto de la sociedad (Higgs y Gilleard, 2015b).

Mientras que el tercer discurso en ningún momento cuestiona la responsabilidad colectiva en la garantía de unos niveles de bienestar satisfactorios para las personas de más edad, el marco de la equidad generacional, en cambio, pretende erosionar la legitimidad de la intervención pública en la atención a los mayores, negando la posibilidad de dar continuidad al contrato intergeneracional. Dicho coloquialmente, el segundo marco echa arena sobre los ojos de las generaciones más jóvenes con la idea fuerza de que sus expectativas de recibir suficiente protección pública en su vejez serán irrealizables.

Incluso, este discurso, en su formulación básica, de clara inspiración neoliberal, incorpora también el argumento, atractivo para sectores de la población progresistas, conforme el cual, en el presente, los recursos públicos dedicados al bienestar de los mayores estarían mejor invertidos en otros grupos de edad. Por este motivo, se hace necesario distinguir en el plano teórico dos variantes del marco discursivo sobre el conflicto entre generaciones que conviven en la discusión pública de distintas sociedades europeas.

Desde luego, es posible defender un reparto distinto de recursos públicos entre generaciones, dando respuesta a otras transformaciones sociales relevantes y a la propia evaluación del rendimiento de los distintos programas de gasto público. Mi preocupación reside en que en la redefinición del modelo social europeo clásico, centrado en la protección de los mayores, no se tomen las precauciones debidas para evitar la contaminación del discurso neoliberal contrario a la idea de responsabilidad pública sobre el bienestar individual, toda vez que, aunque con fines diferentes, se están manejando argumentos próximos, como puede observarse claramente en la tabla 2 . 


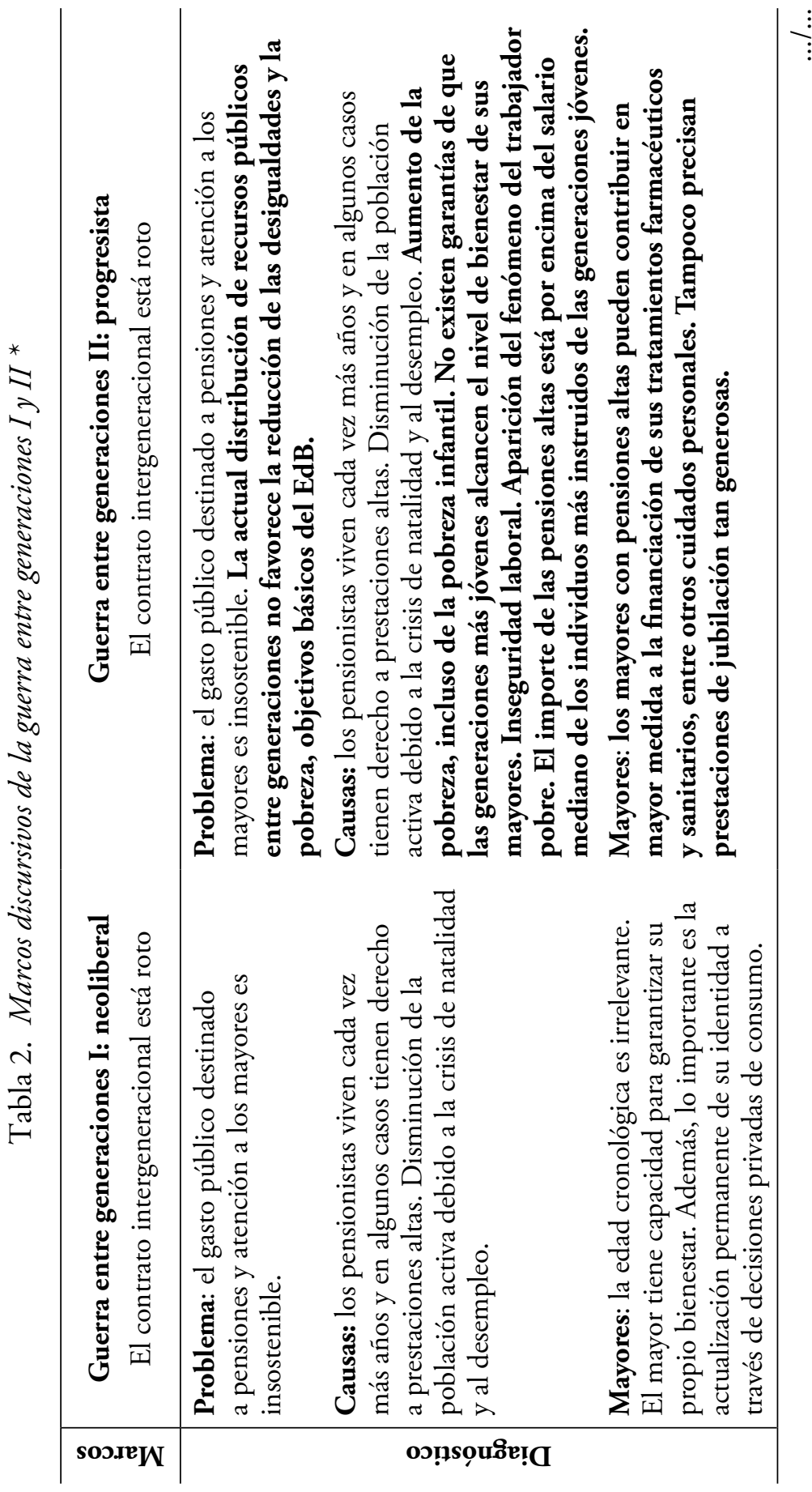




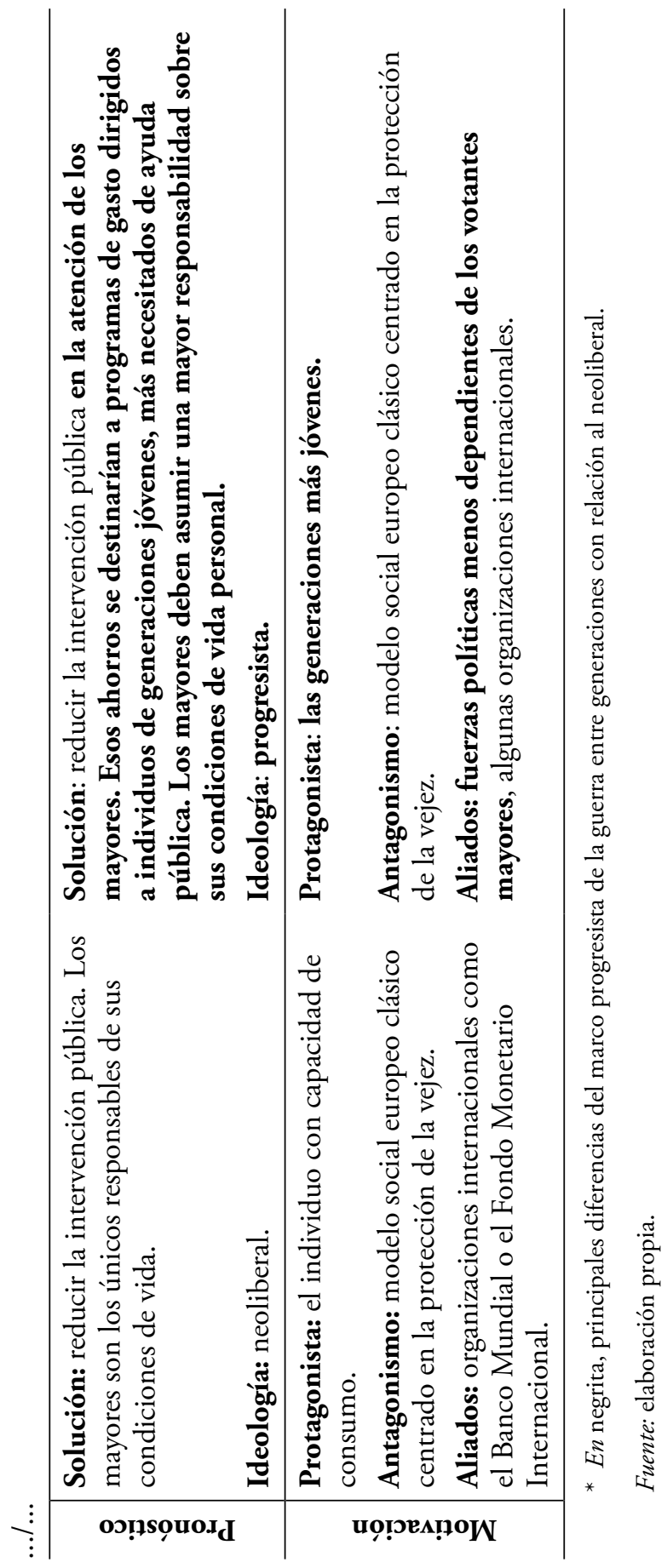


Frente al énfasis en la interdependencia del tercer marco, el segundo discurso de la tabla 1, también en su versión progresista planteada en la tabla 2, abre una batalla entre generaciones por los recursos públicos, más escasos en un contexto de crisis económica y financiera como el abierto en Europa a partir del año 2008. Además de los desequilibrios en las finanzas públicas de algunos países europeos, la propia globalización juega un papel en la cimentación de este marco, por su influencia en el terreno ideológico en la propia construcción de la noción de envejecimiento, la consideración del envejecimiento como riesgo social, y la creación de nuevas estructuras de control y gestión (Phillipson, 2006: 202).

Para terminar este epígrafe, abordo más en profundidad el mito del conflicto o la guerra entre generaciones, principal arma discursiva para deslegitimar la redistribución intergeneracional. Este mito, quiero enfatizar, parece extenderse en la discusión pública europea en este momento, tras la Gran Crisis, casi medio siglo después de haber sido contestado con datos en Estados Unidos, y en oposición a toda la evidencia empírica recabada por la gerontología en sus más de cincuenta ańos de historia.

El lanzamiento de la idea de gerontocracia se produjo en la década de 1970 en Estados Unidos, donde fue propagada por grupos como la Fundación de los Americanos para la Equidad Generacional (Foundation of the Americans for Generational Equity, AGE), una organización financiada por círculos conservadores para promover recortes en los prestaciones sociales a los mayores. Pero, ya en 1974, la Asociación Americana para el Avance de la Ciencia (American Association for the Advancement of Science, AASS) financió un simposio titulado «La década de 1990 y más allá: ¿una gerontocracia?», en el que se abordó directamente la pregunta de si la estadounidense iba a convertirse en una democracia controlada por los mayores. El debate se centró precisamente en las consecuencias políticas del envejecimiento e hizo dialogar diferentes visiones sobre el impacto probable del cambio demográfico en los resultados electorales. Por mucho que aumente el número de personas de 65 ańos o más, la política americana a lo largo del siglo Xxi apenas variará, se concluyó, entonces, con rotundidad (Schulz y Binstock, 2006: 201-202).

El juego de suma cero que plantea el discurso de la justicia generacional implica que los intereses de los jubilados y, en general, de las personas a partir de los 65 años son homogéneos y entran en conflicto con aquellos del resto de generaciones. Todavía más si se acepta la idea del mismo marco de una sociedad en la que la edad es irrelevante frente a la capacidad adquisitiva del individuo - pues un observador con lentes postmodernas vería «una farsa de invariabilidad, de acuerdo con las expectativas sociales» (Biggs, 2006: 115 - _ la realidad es que no hay intereses comunes en este grupo etario que 
puedan imponerse a los jóvenes, sino preferencias diversas entre mayores, tanto o más que entre personas de distintas edades (Busemeyer et al., 2008; Goerres, 2007a y 2009).

Walker ha relacionado ya las siguientes cinco barreras a la participación e influencia política de la tercera edad: 1) no comparten los mismos intereses; 2) la tendencia a la acción colectiva es menor entre las personas retiradas, que suelen autopercibirse como menos eficaces políticamente; 3) aunque hay excepciones, no suelen disponer de canales formales para el ejercicio de la influencia; 4) barreras físicas y mentales; 5) en general no son más conservadores a la hora de votar - lo que puede existir es una tendencia a seguir apoyando al partido por el que han optado siempre- (Walker, 2006: 351-353).

Por lo pronto, las actitudes de las distintas generaciones no tienen relación con la edad cronológica (Kohli et al., 2010). No se dispone de datos consistentes de comportamiento electoral determinado por intereses vinculados a la etapa del ciclo de la vida del votante, o evidencia empírica de movilización política en base a las líneas de un naciente conflicto entre grupos de edad. Entre otras causas, porque existen otras desigualdades sociales más relevantes y por la fortaleza de los lazos familiares y los apoyos instrumentales y/o afectivos mutuos entre generaciones. Si la solidaridad intergeneracional sigue gozando de buena salud a nivel micro (Bengtson y Putney, 2006; Kohli et al., 2010; Kohli, 2015), parece poco probable que surja una dinámica opuesta a nivel macro.

Nada de lo anterior quiere decir que no exista potencial para un incremento de la movilización política en torno a líneas generacionales en el futuro, que los politólogos también debemos tratar de prever y, en el que caso de que se produzca, explicar. Pero, en definitiva, a día de hoy, los jóvenes pueden dormir tranquilos, o «dejar de preocuparse y querer a los pensionistas» (Lynch, 2015: 53).

\section{CONCLUSIONES}

A pesar de los problemas presupuestarios que están condicionando las decisiones de gasto en algunos Estados miembros de la UE, hasta la fecha, el envejecimiento no parece haber abierto una competencia entre generaciones por los recursos públicos ni provocado la apertura de una nueva línea de conflicto político basada en la edad, entre otras causas, porque los intereses de los mayores son diversos y la solidaridad a nivel micro muy resistente. Sin embargo, en el debate público de algunas sociedades europeas está cada vez más presente el mito de la gerontocracia o del creciente control de la democracia por parte de las personas de más edad.

Recuerdo que el fantasma de la gerontocracia se puso en circulación en Estados Unidos en la década de 1970 por parte de colectivos interesados en 
recortar las prestaciones sociales de los mayores. Inmediatamente, fue contestado con teorías y datos. Esta falsa creencia desembarcó primero en el Reino Unido y, sobre todo desde los años 1990, también en Europa continental. A pesar de tener en contra toda la evidencia empírica de más de medio siglo de gerontología, es ahora, tras la Gran Recesión iniciada en 2008, cuando empieza a tener éxito.

Un contrato intergeneracional viable, tanto a nivel micro como macro, es un elemento común a cualquier sociedad humana, y debe estar abierto a discusión y cambio. Seguramente, en este momento, las generaciones más jóvenes de algunas sociedades envejecidas precisen una asignación de recursos públicos mayor, en beneficio de la productividad de sus economías y, sobre todo, por razones de equidad. No obstante, convendría que ninguna opinión pública europea dejase de creer que el bienestar de sus séniores es una responsabilidad colectiva, por influencia no del supuesto poder de estos sino de estrategias discursivas que solo interesan a aquellos contribuyentes que prefieren dejar de pagar lo que por capacidad les corresponde, en sistemas fiscales más o menos progresivos, sostén del modelo social europeo.

Defiendo que la ciencia política tiene pocas tareas más relevantes en su agenda que aportar ideas y datos al debate sobre los efectos políticos del envejecimiento. Se necesitan mejores preguntas y más evidencia para responder, entre otras muchas, a cuestiones como: ¿cuándo los partidos modifican su oferta electoral en una sociedad envejecida? ¿Algunas fuerzas políticas se están apropiando de temas como las pensiones o la atención a la dependencia? ¿El aumento del porcentaje de mayores entre los afiliados a los partidos afecta a sus prioridades y posiciones? ¿Qué explica la variación en el diseño, ejecución e impacto de políticas de envejecimiento?

Se precisan, asimismo, desarrollos teóricos sobre las dinámicas políticas de las sociedades envejecidas, que asistan en la construcción de datos comparables, así como en la comprensión de las observaciones empíricas y sus implicaciones normativas.

Finalmente, como se está haciendo ya, se debe mostrar disponibilidad para contribuir a mejorar las iniciativas políticas orientadas a facilitar la participación de los mayores, así como actualizar el contrato generacional basado en la idea de responsabilidad colectiva sobre el bienestar de los ciudadanos de todas las edades, con el objetivo de mantener su viabilidad.

\section{Bibliografía}

Alstott, A. L. (2016). A New Deal for Old Age. Toward a Progressive Retirement. Cambridge (MA); London:HarvardUniversityPress.Disponibleen:https://doi.org/10.4159/9780674545816.

Banco Mundial (1994). Averting the Old Age Crisis. A World Bank Policy Research Report. New York: Oxford University Press. 
Bauman, Z. (2007). Liquid Times: Living in an Age of Uncertainty. Cambridge: Polity.

Beck, U. (1992). The Risk Society. London: Sage Books.

Bengtson, V. L., Putney, N. M. y Johnson, M. L. (2005). The problem of theory in gerontology today. En M. L. Johnson (ed.). The Cambridge Handbook of Age and Aging (pp. 3-20). Cambridge: Cambridge University Press. Disponible en: https://doi.org/10.1017/ CBO9780511610714.003.

Bengtson, V. L. y Putney, N. M. (2006). Future 'Conflicts' across Generations and Cohorts? En J. A. Vincent, C. R. Phillipson y M. Downs. The Futures of Old Age (pp. 20-29). London: Sage. Disponible en: https://doi.org/10.4135/9781446211533.n2.

Biggs, S. (2006). Ageing Selves and Others: Distinctiveness and Uniformity in the Struggle for Intergenerational Solidarity. En J. A. Vincent, C. R. Phillipson y M. Downs. The Futures of Old Age (pp. 109-116). London: Sage. Disponible en: https://doi.org/10.1017/ S0144686X06264894.

Binstock, R. H. (1972). Interest-Group Liberalism and the Politics of Aging. The Gerontologist, 12, 265-280. Disponible en: https://doi.org/10.1093/geront/12.3_Part_1.265.

- (1991). From the great society to the aging society: 25 years of the older Americans Act. Generations, 15 (3), 11-18.

- y George, L. K. (2006). Handbook of the Aging and the Social Sciences (6. ${ }^{\mathrm{a}} \mathrm{ed}$.). Amsterdam: Academic Press. Disponible en: https://doi.org/10.1016/B9-78-012088-3/88250-0031.

Bristow, J. (2015). Baby Boomers and Generational Conflict. Basingstoke: Palgrave. Disponible en: https://doi.org/10.1057/9781137454737.

Busemeyer, M. R., Goerres, A. y Weschle, S. (2008). Demands for Redistributive Policies in an Era of Demographic Aging: The Rival Pressures from Age and Class in 15 OECD Countries. Max Planck Institute for the Study of Societies (MPIFG). Discussion Paper DP08, 3.

Comisión Europea (2005). Libro verde Frente a los cambios demográficos, una nueva solidaridad entre generaciones [Bruselas, 16/03/2005, COM (2005) 94 final].

Cumming, E. y Henry, W. E. (1961). Growing old, the process of disengagement. New York: Basic Books.

Cutler, N. E. (1977). Demographic, Social-Psychological, and Political Factors in the Politics of aging: A Foundation for Research in 'Political Gerontology'. American Political Science Review, 71 (3), 1011-1025. Disponible en: https://doi.org/10.2307/1960104.

Day, C. L. (1990). What Older Americans Think: Interest Groups and Aging Policy. Princeton: Princeton University Press. Disponible en: https://doi.org/10.1515/9781400861125.

Doyle, M. (2015). The politics of old age. Older people's interest organisations and collective action in Ireland. Manchester: Manchester Univeristy Press. Disponible en: https://doi. org/10.7228/manchester/9780719090479.003.0002.

Eurostat (2011). Active Ageing and Solidarity between Generations. A Statistical Portrait of the European Union 2012. Luxemburg: Oficina de Publicaciones de la UE.

Fennell, G., Phillipson, C. y Evers, H. (1988). The Sociology of Old Age. Milton Keynes: Open University Press. Disponible en: https://doi.org/10.1017/S0144686X0000711X.

George, L. (2006). Perceived Quality of Life. En R. H. Binstock y L. K. George. Handbook of the Aging and the Social Sciences (6. ${ }^{\mathrm{a}}$ ed.) (pp. 320-336). Amsterdam: Academic Press. Disponible en: https://doi.org/10.1016/B978-012088388-2/50021-3. 
Giddens, A. (1991). Modernity and Self-Identity. Cambridge: Polity Press.

Goerres, A. (2007a). Can We Reform the Welfare State in Times of 'Grey' Majorities? The Myth of an Electoral Opposition between Younger and Older Voters in Germany. Max Planck Institute for the Study of Societies. Working Paper, 5.

— (2007b). Why are Older People More Likely to Vote? The Impact of Ageing on Electoral Turnout in Europe. British Journal of Politics and International Relations, 9, 90-121. Disponible en: https://doi.org/10.1111/j.1467-856x.2006.00243.x.

(2008). The grey vote: Determinants of older voters' party choice in Britain and West Germany. Electoral Studies, 27, 285-304. Disponible en: https://doi.org/10.1016/j. electstud.2007.12.007.

(2009). The Political Participation of Older People in Europe. The Greying of our Democracies. Basingstoke: Palgrave. Disponible en: https://doi.org/10.1057/9780230233959.

Held, D. y McGrew, A. (2002). Governing Globalization: Power, Authority and Global Governance. Cambridge: Polity Press.

Higgs, P. y Gilleard, C. (2015a). Generational Justice, Generational Habitus and the 'Problem' of the Baby Boomers. En C. Torp (ed.). Challenges of Aging. Pensions, Retirement and Generational Justice (pp. 251-263). Basingstoke: Palgrave. Disponible en: https://doi. org/10.1057/9781137283177_13.

— (2015b). Rethinking Old Age. Theorising the Fourth Age. Basingstoke: Palgrave. Disponible en: https://doi.org/10.1007/978-1-137-38400-3.

Hudson, R. B. (2016). Politics and Policies of Afing in the United States. En L. K. George y K. F. Ferraro (eds.). Handbook of Aging and the Social Sciences (6. ${ }^{a}$ ed.) (pp. 441-459). London, San Diego y Waltham: Elsevier.

Kohli, M. (2015). Generations in Aging Societies: Inequalities, Cleavages, Conflicts. En C. Torp (ed.). Challenges of Aging. Pensions, Retirement and Generational Justice (pp. 265-288). Basingstoke: Palgrave. Disponible en: https://doi.org/10.1057/9781137283177_14.

- et al. (2010). Linkages among Adult Family Generations: Evidence from Comparative Survey Research. En P. Heady y M. Kohli (eds). Famili, Kinship and State in Contemporary Europe, vol. 3: Perspectives on Theory and Policy (pp. 195-220). Frankfurt: Campus.

Kolb, P. (2014). Understanding Aging and Diversity. London-New York: Routledge.

Lynch, J. (2006). Age in the Welfare State. The Origins of Social Spending on Pensioners, Workers, and Children. Cambridge: Cambridge University Press. Disponible en: https://doi. org/10.1017/CBO9780511606922.

- (2015). Age Politics and Pension Systems Development and Reform. En C. Torp (ed.). Challenges of Aging. Pensions, Retirement and Generational Justice (pp. 49-60). Basingstoke: Palgrave. Disponible en: https://doi.org/10.1057/9781137283177_4.

Macnicol, J. (2015). Neoliberalising Old Age. Cambridge: Cambridge University Press. Disponible en: https://doi.org/10.1017/CBO9781316335666.

Minkler, M. y Robertson, A. (1991). The ideology of age/race wars: Deconstructing a social problem. Ageing and Society, 11, 1-23. Disponible en: https://doi.org/10.1017/ S0144686X00003809.

— y Fadem, P. (2002). Successful Aging: A disability perspective. Journal of Disability Policy Studies, 12, 229-235. Disponible en: https://doi.org/10.1177/104420730201200402. 
Moreno, L. (2013). La Europa asocial. Barcelona: Península. Disponible en: https://doi. org/10.1093/europace/eut032.

Naciones Unidas (2003). Declaración Política y Plan de Acción Internacional de Madrid sobre el Envejecimiento. New York.

Natali, D. (2012). The politics of the new welfare state. Oxford: Oxford University Press.

Organización Mundial de la Salud (2002). Active Ageing: A Policy Framework. Disponible en: https://goo.gl/T9GoLs.

Organización para la Cooperación y el Desarrollo Económicos (1988). Ageing populations The social policy implications. Paris: OCDE.

- (1998). Maintaining prosperity in an aging society. Paris: OCDE.

- (2001). Aging and income. Paris: OCDE.

Phillipson, C. (2006). Ageing and Globalization. En J. A. Vincent, C. R. Phillipson y M. Downs. The Futures of Old Age (pp. 201-207). London: Sage. Disponible en: https:// doi.org/10.4135/9781446211533.n20.

Quadagno, J. (1989). Generational equity and the politics of the welfare state. Politics and Society, 17, 353-376. Disponible en: https://doi.org/10.1177/003232928901700303.

Rowe, J. W. y Kahn, R. L. (1987). Human Aging: Usual and Successful. Science, 237 (4811), 143-149. Disponible en: https://doi.org/10.1126/science.3299702.

- (1998). Successful Aging. New York: Pantheon Books.

Schulz, J. H. y Binstock, R. H. (2006). Aging Nations: The Economics and Politics of Growing Older in America. Westport: Praeger.

Sidorenko, A. y Walker, A. (2004). The Madrid International Plan of Action on Ageing: from conception to implementation. Ageing and Society, 24 (1), 147-165. Disponible en: https://doi.org/10.1017/S0144686X03001661.

Torp, C. (ed.). (2015). Challenges of Aging. Pensions, Retirement and Generational Justice. Basingstoke: Palgrave. Disponible en: https://doi.org/10.1057/9781137283177.

Vera-Sanso, P. et al. (2014). Participation and social connectivity. En A. Walker (ed.). The new science of ageing (pp. 181-2018). Bristol-Chicago: Policy Press. Disponible en: https:// doi.org/10.1332/policypress/9781447314660.003.0006.

Vincent, J. (1996). Who's afraid of an aging population? Nationalism, the free market and the construction of old age as an issue, Critical Social Policy, 16, 3-26. Disponible en: https://doi.org/10.1177/026101839601604701.

Vincent, J. A., Phillipson, C. R. y Downs, M. (2006). The Futures of Old Age. London: Sage. Disponible en: https://doi.org/10.4135/9781446211533.n19.

Walker, A. (1990). The economic 'burden' of aging and the prospect of intergenerational conflict. Ageing and Society, 10 (4), 377-396. Disponible en: https://doi.org/10.1017/ S0144686X00007376.

- (2006). Aging and Politics: An International Perspective. En R. H. Binstock y L. K. George (eds.) ( $6^{2}$ ed). Handbook of the Aging and the Social Sciences (pp. 339-359). Amsterdam: Academic Press.

- (2014). A new policy perspective on ageing. En A. Walker (ed.). The new science of ageing (pp. 241-259). Bristol-Chicago: Policy Press. Disponible en: https://doi. org/10.2307/j.ctt1t88xdh.12. 
- y Naegele, G. (2009). Major Policy Challenges of Ageing Societies: Britain and Germany Compared. En A.Walker y G. Naegele (eds.). Social Policy in Ageing Societies. Britain and Germany Compared (pp. 1-21). Basingstoke: Palgrave.

Westerhof, G. J. y Tulle, E. (2007). Meaning of ageing and old age: Discursive contexts, social attitudes and personal identities. En J. Bond, S. Peace, F. Dittmann-Kohli y G. Westerhof. Ageing in Society (pp. 235-254). London: Sage. Disponible en: https://doi. org/10.4135/9781446278918.n11.

Williamson, J. B. y Béland, D. (2016). The Future of Retirement Security in Comparative Perspective. En L. K. George y K. F. Ferraro (eds.). Handbook of Aging and the Social Sciences (6a ed.) (pp. 461-481). London-San Diego-Waltham: Elsevier. Disponible en: https://doi.org/10.1016/B978-0-12-417235-7.00022-6. 
Anexo

Tabla AI. Esperanza de vida al nacer (en 2015) y porcentaje de mayores (en 2016) en los Estados miembros de la UE

\begin{tabular}{|c|c|c|c|}
\hline & $\begin{array}{c}\text { Esperanza de vida } \\
\text { al nacer de los } \\
\text { hombres* }\end{array}$ & $\begin{array}{c}\text { Esperanza de vida } \\
\text { al nacer de las } \\
\text { mujeres* }\end{array}$ & $\begin{array}{c}\text { Porcentaje de la } \\
\text { población de } 65 \\
\text { o más ańos }\end{array}$ \\
\hline Media UE & $77,9(62,6)$ & $83,3(63,3)$ & 19,2 \\
\hline Alemania & $78,3(65,3)$ & $83,1(67,5)$ & 21,1 \\
\hline Austria & $78,8(57,9)$ & $83,7(58,1)$ & 18,5 \\
\hline Bélgica & $78,7(64,4)$ & $83,4(64)$ & 18,2 \\
\hline Bulgaria & $71,2(61,5)$ & $78,2(65)$ & 20,4 \\
\hline Chipre & $79,9(63,1)$ & $83,7(63,4)$ & 15,1 \\
\hline Croacia & $74,4(55,3)$ & $80,5(56,8)$ & 19,2 \\
\hline Dinamarca & $78,8(60,4)$ & $82,7(57,6)$ & 18,8 \\
\hline Eslovaquia & $73,1(54,8)$ & $80,2(55,1)$ & 14,4 \\
\hline Eslovenia & $77,8(58,5)$ & $83,9(57,7)$ & 18,4 \\
\hline España & $80,1(63,9)$ & $85,8(64,1)$ & 18,7 \\
\hline Estonia & $73,2(53,8)$ & $82,2(56,2)$ & 19 \\
\hline Finlandia & $78,7(59,4)$ & $84,4(56,3)$ & 20,5 \\
\hline Francia & $79,2(62,6)$ & $85,5(64,6)$ & 18,8 \\
\hline Grecia & $78,5(63,9)$ & $83,7(64,1)$ & 21,3 \\
\hline Hungría & $72,3(58,2)$ & $79(60,1)$ & 18,3 \\
\hline Irlanda & $79,6(66,6)$ & $83,4(67,9)$ & 13,2 \\
\hline Italia & $80,3(62,6)$ & $84,9(62,7)$ & 22 \\
\hline Letonia & $69,7(51,8)$ & $79,5(54,1)$ & 19,6 \\
\hline Lituania & $69,2(54,1)$ & $79,7(58,8)$ & 19 \\
\hline Luxemburgo & $80(63,7)$ & $84,7(60,6)$ & 14,2 \\
\hline Malta & $79,7(72,6)$ & $84(74,6)$ & 19 \\
\hline Países Bajos & $79,9(61,1)$ & $83,2(57,2)$ & 18,2 \\
\hline
\end{tabular}




\begin{tabular}{lccc}
...... & $\begin{array}{c}\text { Esperanza de vida } \\
\text { al nacer de los } \\
\text { hombres* }\end{array}$ & $\begin{array}{c}\text { Esperanza de vida } \\
\text { al nacer de las } \\
\text { mujeres* }\end{array}$ & $\begin{array}{c}\text { Porcentaje de la } \\
\text { población de 65 } \\
\text { o más ańos }\end{array}$ \\
\hline Polonia & $73,5(60,1)$ & $81,6(63,2)$ & 16 \\
\hline Portugal & $78,1(58,2)$ & $84,3(55)$ & 20,7 \\
\hline Suecia & $80,4(74)$ & $84,1(73,8)$ & 19,8 \\
\hline República Checa & $75,7(62,4)$ & $81,6(63,7)$ & 18,5 \\
\hline Reino Unido & $79,2(63,7)$ & $82,8(63,3)$ & 17,9 \\
\hline Rumanía & $71,5(59)$ & $78,7(59,4)$ & 17,4 \\
\hline
\end{tabular}

* Entre paréntesis, el indicador ańos de vida saludable al nacer, definida como la ausencia de limitaciones a la autonomía personal.

Fuente: elaboración propia con datos de Eurostat. 


\section{Glosario}

Contrato intergeneracional: expectativas y obligaciones compartidas en relación al envejecimiento y la sucesión de generaciones en el tiempo en una estructura social.

Equidad o justicia generacional: etiqueta trampa de un marco discursivo, puesto en circulación en la década de 1970 en Estados Unidos, contrario al Estado de bienestar. Trata de difundir la falsa creencia de que el contrato intergeneracional, en el que se fundamenta el modelo social europeo clásico, está roto o es insostenible en un nuevo escenario globalizado y envejecido.

Gerontología: campo interdisciplinar de estudio del envejecimiento de la población que comienza a desarrollarse en los años 1960. Inicialmente, se centró en temas de ciencias de la salud. Con el paso del tiempo, fue incorporando cuestiones propias de las ciencias sociales y las humanidades.

Gerontocracialsenior power/grey power: control del proceso político democrático por parte de los séniores con el objetivo de imponer sus intereses, favorecido por el porcentaje creciente de personas mayores sobre el total de electores de un país y su menor abstencionismo. Los datos demuestran que es un mito.

Tercera edad: sinónimo de vejez. Véase vejez. Con el aumento de la esperanza de vida y las mejoras en las condiciones físicas y cognitivas de los adultos mayores, se ha empezado a distinguir entre tercera y cuarta edad. Esta última es la fase final del ciclo vital, a la que accede un individuo cuando empeora su salud hasta el punto de perder su autonomía.

Vejez: período cronológico diferenciado de otras etapas de la vida, como la niñez, la adolescencia, la juventud o la madurez. El criterio más habitual para marcar el tránsito a la vejez es la edad de 65 años. El postmodernismo y el neoliberalismo son contrarios a reconocer etapas vitales. Para las personas neoliberales las diferencias entre individuos no vienen dadas por la edad cronológica sino por su capacidad adquisitiva y sus hábitos de consumo. 